STATE OF ALASKA

DEPARTMENT OF NATURAL RESOURCES

DIVISION OF GEOLOGICAL AND GEOPHYSICAL SURVEYS

Guy R. Martin - Commissioner

Ross G. Schaff - State Geologist

August 1975

This report is preliminary and has not been edited or reviewed for conformity with Alaskg Division of Geological and Geophysical Surveys standards.
Alaska Open File Report 83 RADIOMETRIC SAGE MAP OF AZASKA ALEUTIAN ISLANDS

by

Frederlc H. Wilson and Donald L. Turner 


\section{RADIOMETRIC AGE MAP OF ALASKA}

\section{by}

Frederic H. Wilson ${ }^{1}$ and Donald L. Turner ${ }^{2}$

This map includes published, thesis, and open-file radiometric data available to us as of June, 1975. Some dates are not plotted because of inadequate location data in the original references.

The map is divided into five sections, based on 1:1,000,000 scale enlargements of the National Atlas maps of Alaska. Within each section (e.g., southeastern Alaska), radiometric dates are plotted and keyed to $1: 250,000$ scale quadrangles. Accompanying each map section is table 1, listing map numbers and the sample identification numbers used in DGGS Special Report 10: Radiometric Dates from Alaska--A 1975 Compilation $^{3}$. The reader is referred to Special Report 10 for more complete information on location, rock type, dating method, and literature references for each age entry. A listing of dates in Special Report 10 which require correction or deletion is included as table 2. Corrected and additional entries are listed in table 3 . The listings in tables 2 and 3 follow the format of Special Report 10 . Table 4 is a glossary of abbreviations used for quadrangle name, rock type, mineral dated, and type of dating method used.

The authors wish to thank the Alaskan Geology Branch of the U.S. Geological Survey for partial support of this project. Thanks are also extended to F.H. Weber of the U.S. Geological Survey, R.B. Forbes of the University of Alaska Geophysical Institute and Geology Dept., and T.E. Smith of DGGS for their assistance and encouragement.

1. U.S. Geological Survey, College, AK., 99701.

2. Geophysieal Institute and Geology Dept., Untv. of Alaska, Fairbanks, 99701.

3. Turner, D.L., Grybeck. D.G., and Wilson, F.H., 1975, Radiometric dates from Alaska---A 1975 compilation. Alaska Div. of Geol. and Geophys. Surveys Spec. Rept. 10. (Available from Álaska OGGS, Box 80007, College. AK., 99701, 51.00. ) 
Table 1. Map numbers and corresponding identification numbers used in Special Report 10; 11sted by 1:250,000 scale quadrangle - Aleutian Islands.

ADAK

Nap

1

3

4

6

7

8

9

Map

1

2

ATTU

Map

1

2

PORT MOLLER

Map

1
2
2
PRIBILOF ISLANDS

Compilation

$$
\text { P67 }
$$

P 35

P38

P5

P52

P66

P 10

P 11

P12

P13

P15

P19

P2

$\mathrm{P} 20$

P21

ATKA 1

ATKA 2

Compilation

3 (ATTU 3)

6 (ATTL! 6)

Compilation

AA76 (405M)

AA77 (406M)

AA77, (406B)

RAT ISLANDS
Map Compilation

AA49 (69AMC-15)

AA50 (69AMC-17)

AA51 (69AMC-2)

AA52 (69AMC-11)

AA46 (iJJC 87A 66)

An47 (69A1C-12)

AA48 (W.JC-1-67)
UATAK

Corpllat1on

ASH1

ASH2

$\mathrm{CCR}$ 


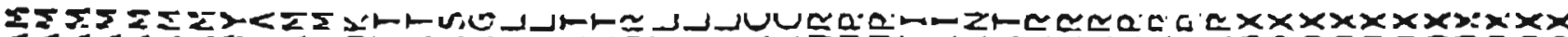

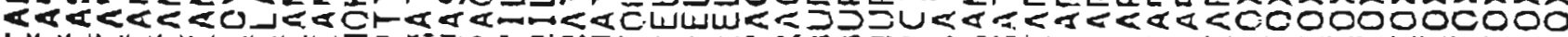
UUUUUUU UUUIa

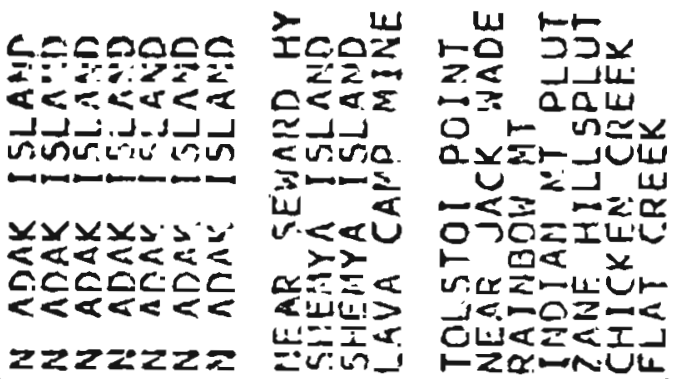

UU⿺𠃊II

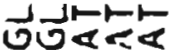
$x y$. जon

Uบizzz

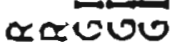
드난

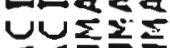
$<<$

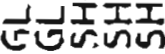

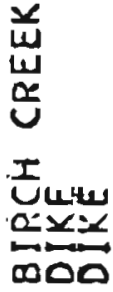
.

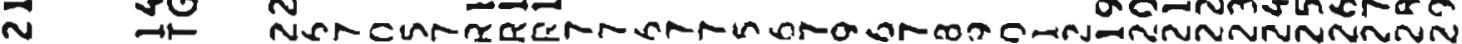

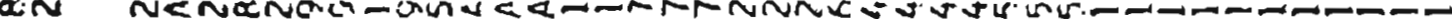

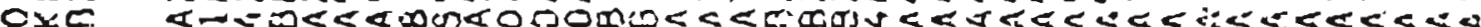

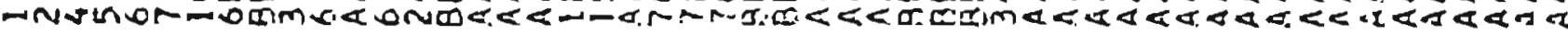

잉요

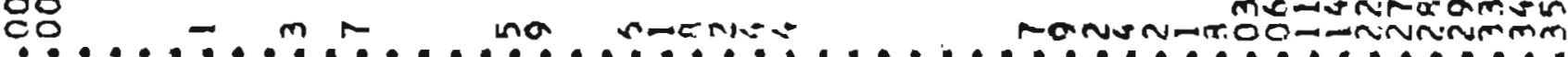

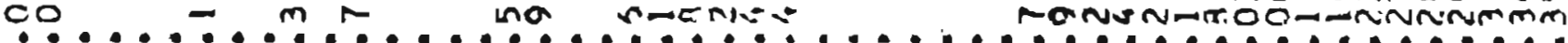

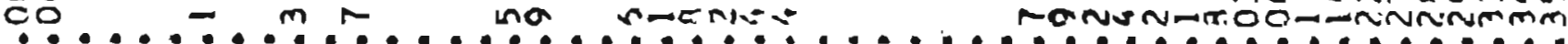

mententamisin

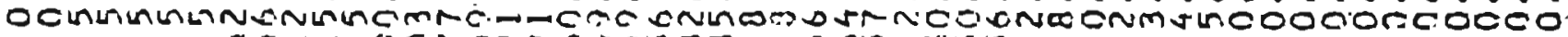

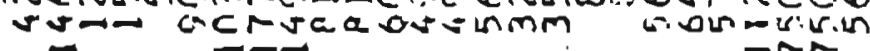
-

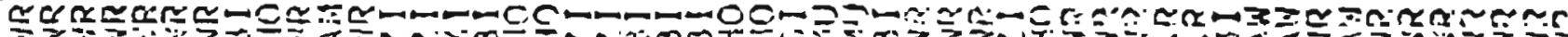

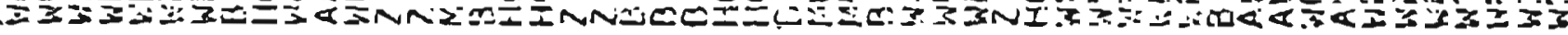

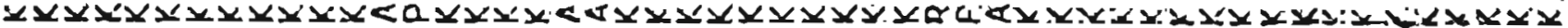

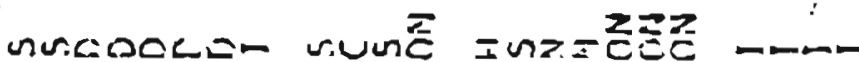

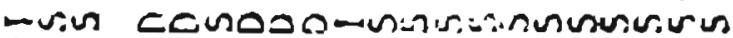

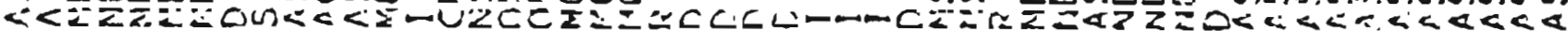

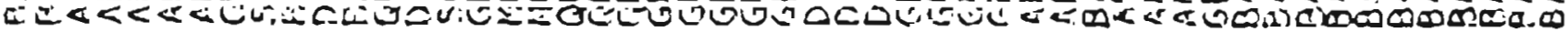

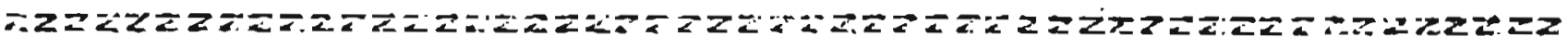

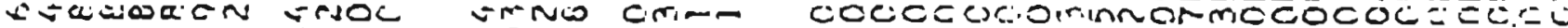

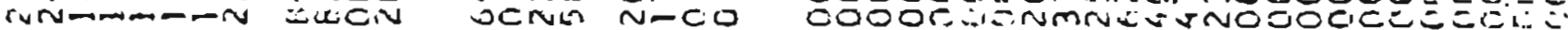

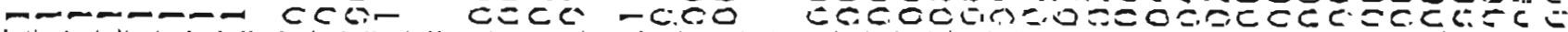

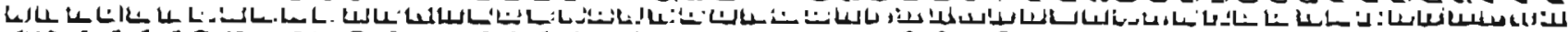

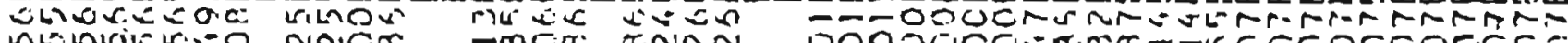

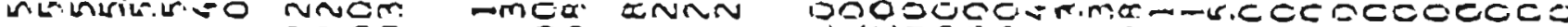

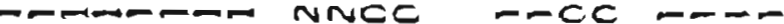

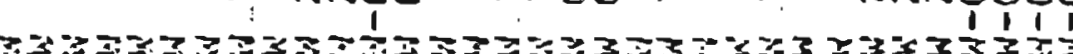

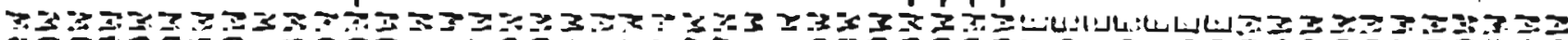

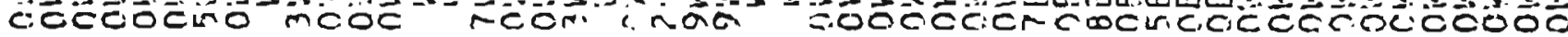
$\ldots \ldots \ldots$

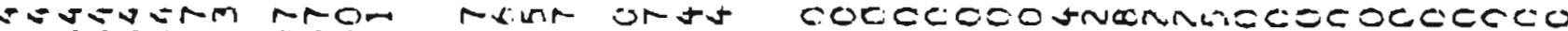

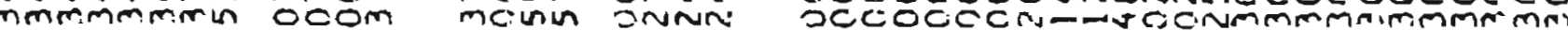

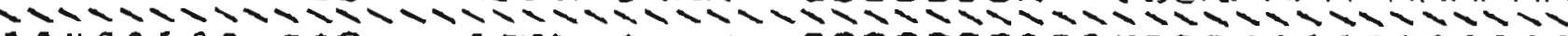

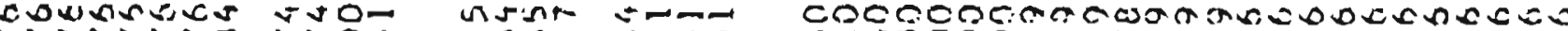

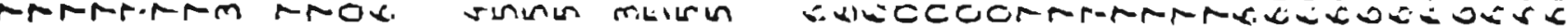

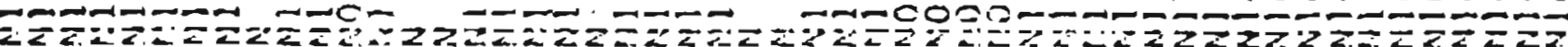

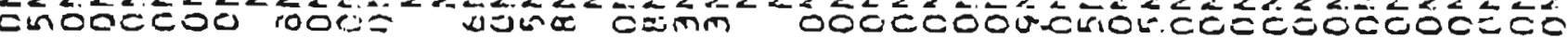

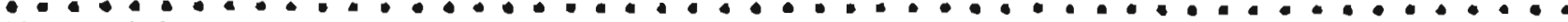

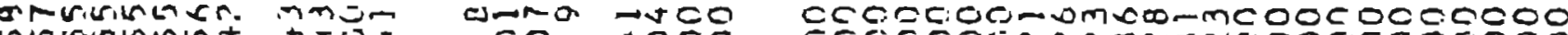

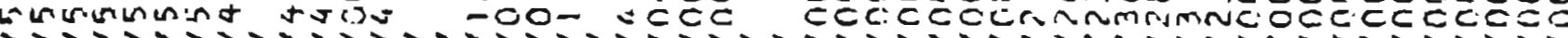

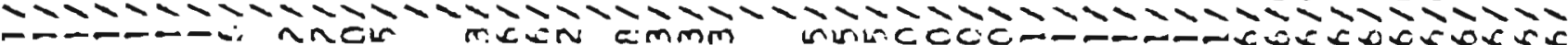

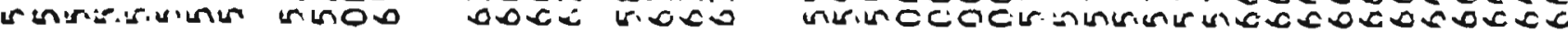

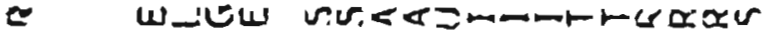

rorigrisnis:

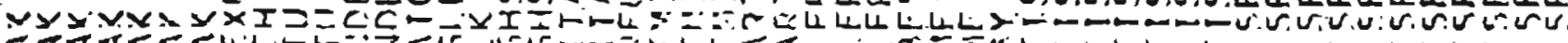

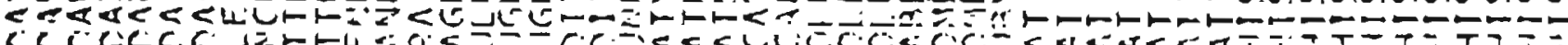

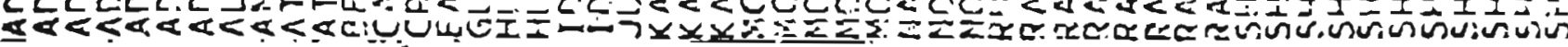




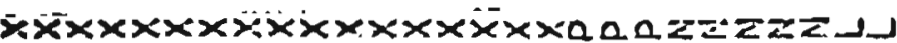
00000000000000000<

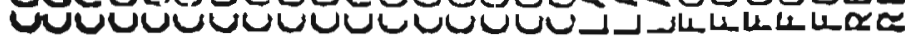

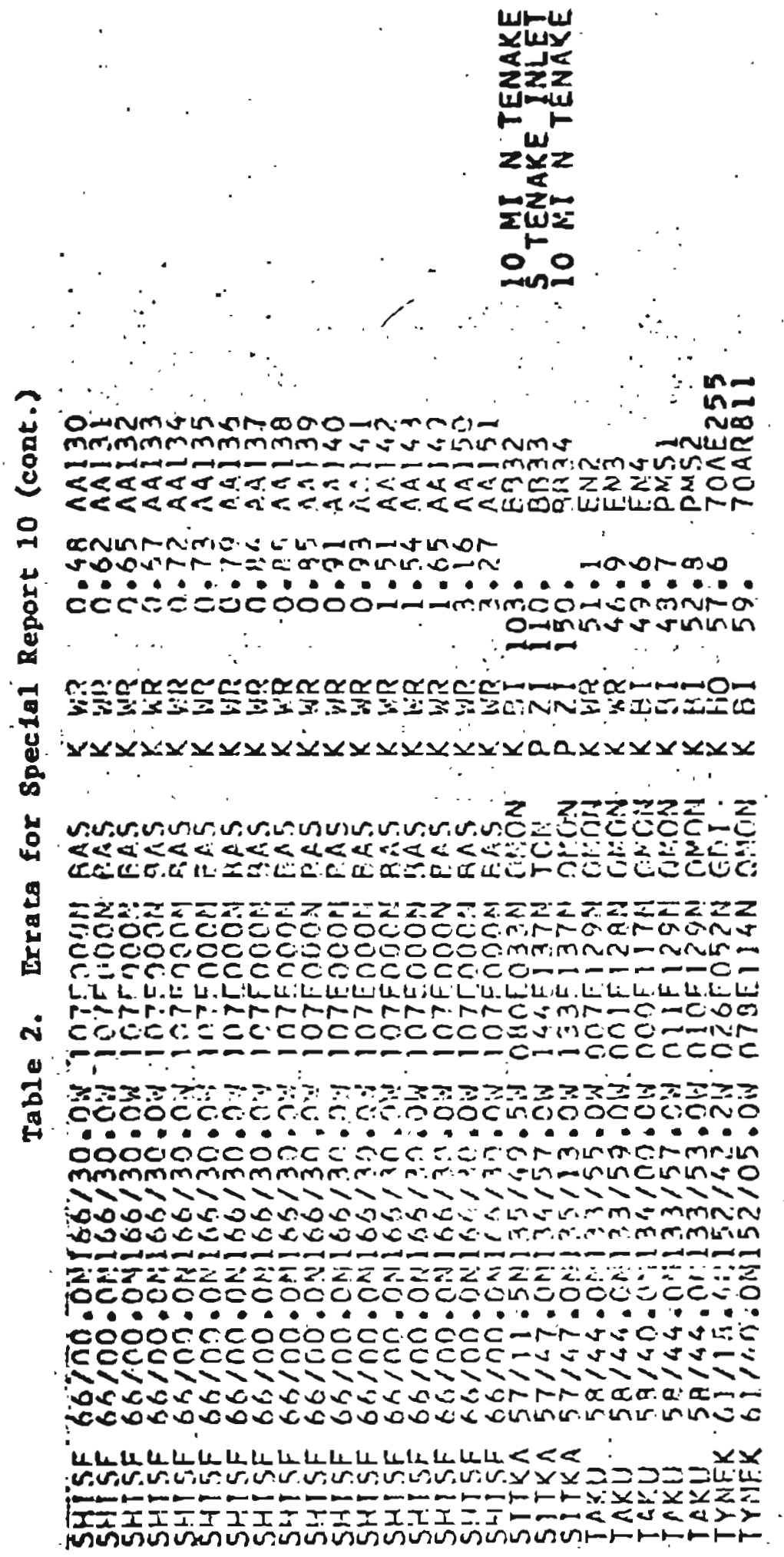


3.

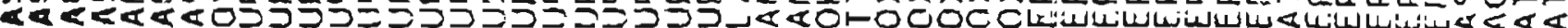

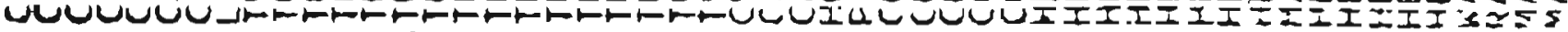

cococol

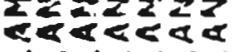

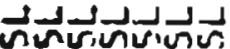

$x \times y \geq x x y$

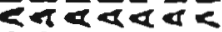
c.e.ciche <<<<\&<<

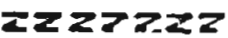

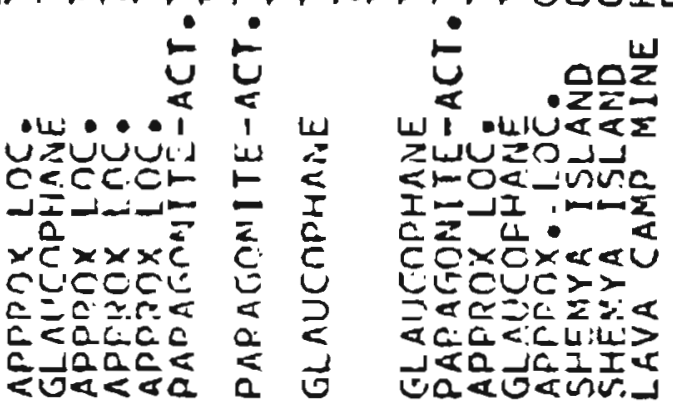

w 2 . 
YUi

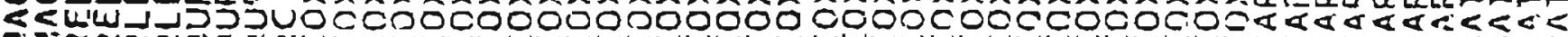

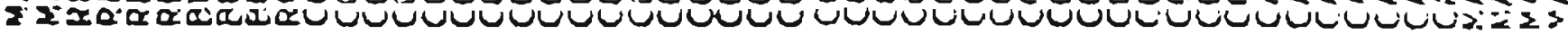

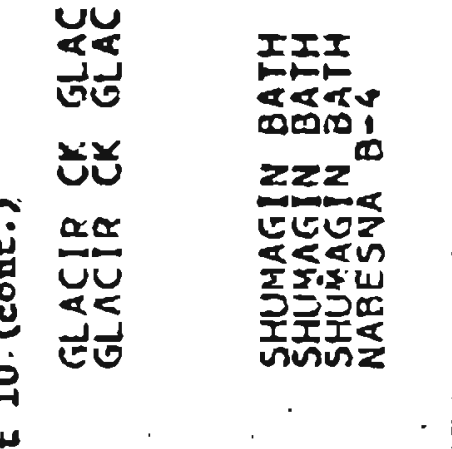

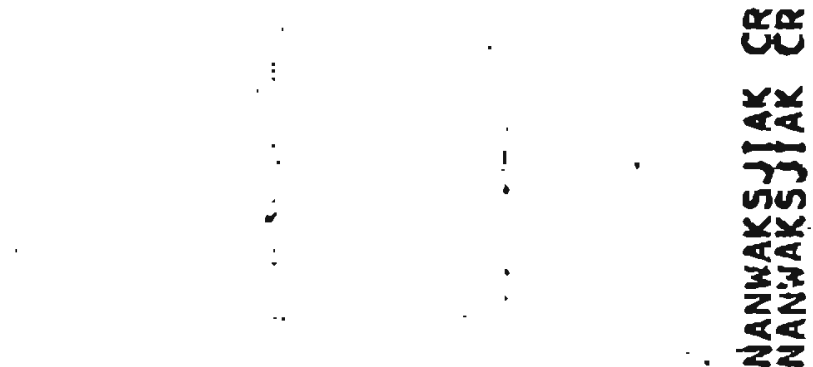

Q10

$\ln 000$

NกN

$\rightarrow \rightarrow-1$

rrararason

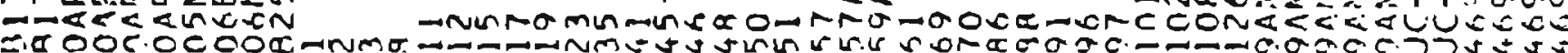
mEr

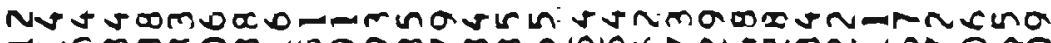

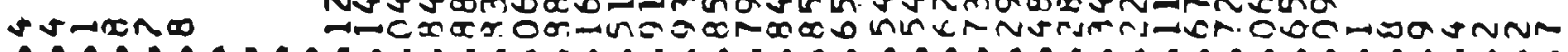

$\cdots$

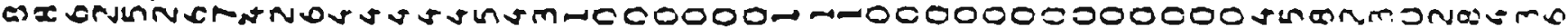

nimmenino-

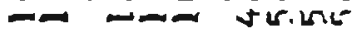

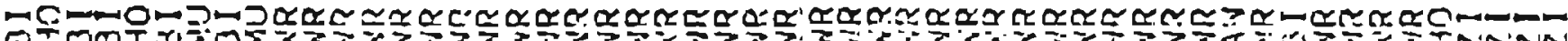

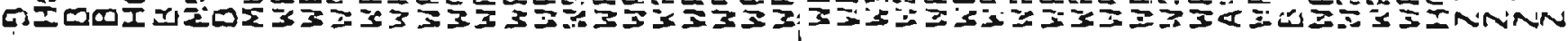

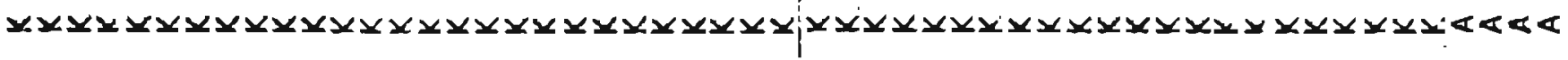

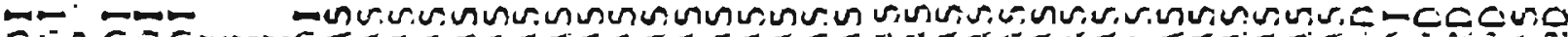

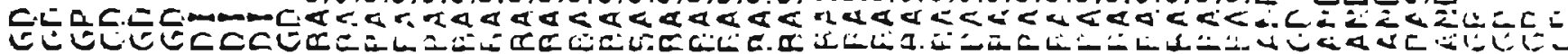

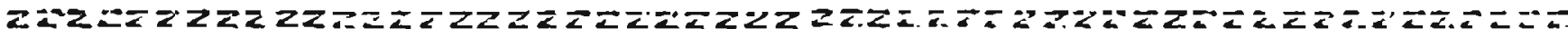

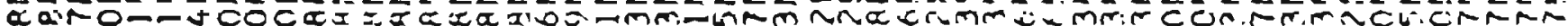

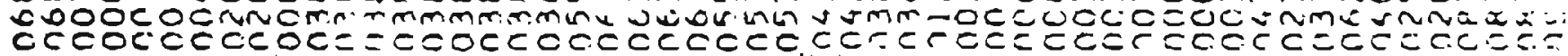

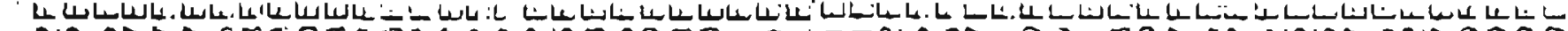

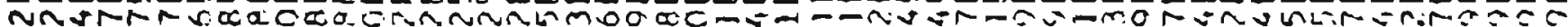
으.nกna

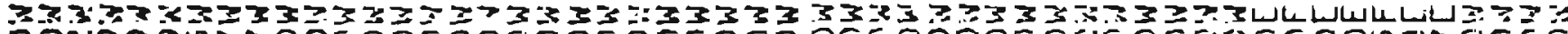

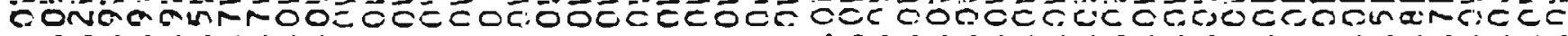

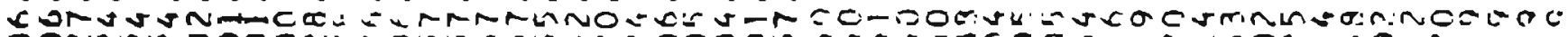

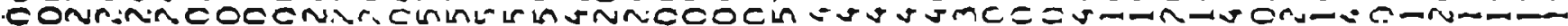

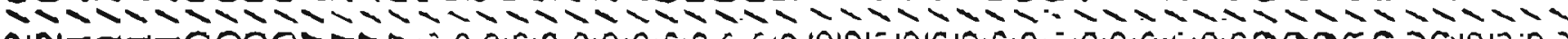

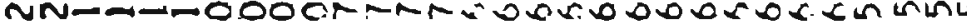

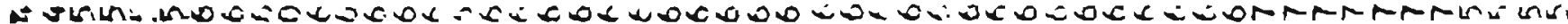

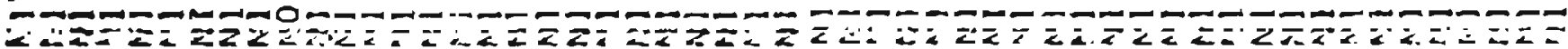

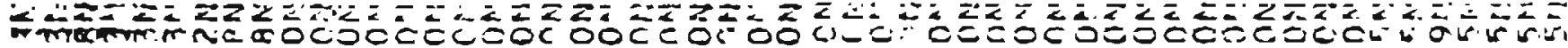

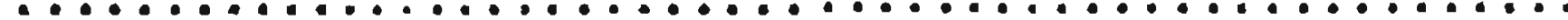

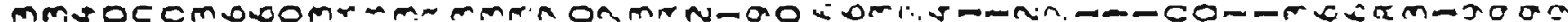

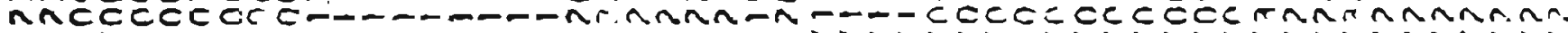

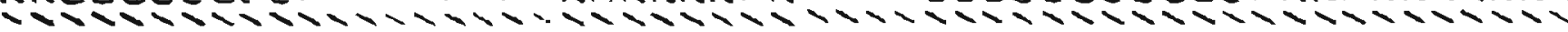

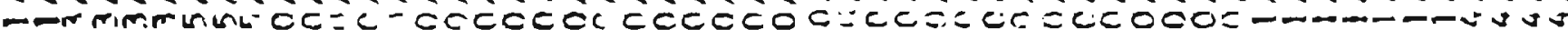
On

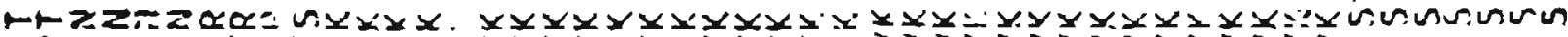

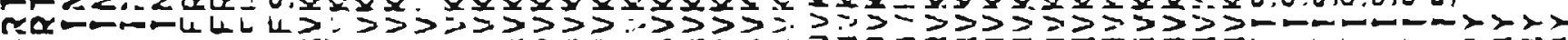

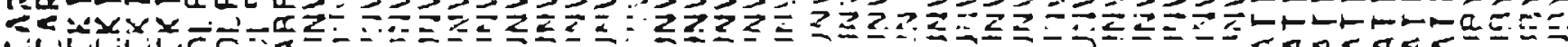

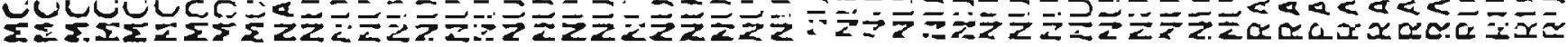




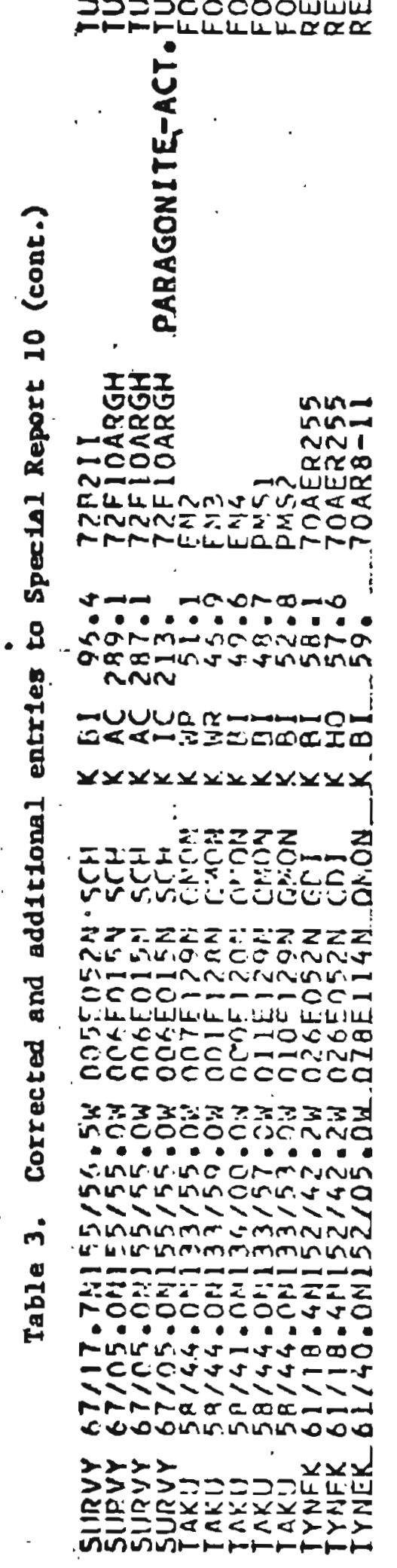


Table 4. Comp1lation glogeary.

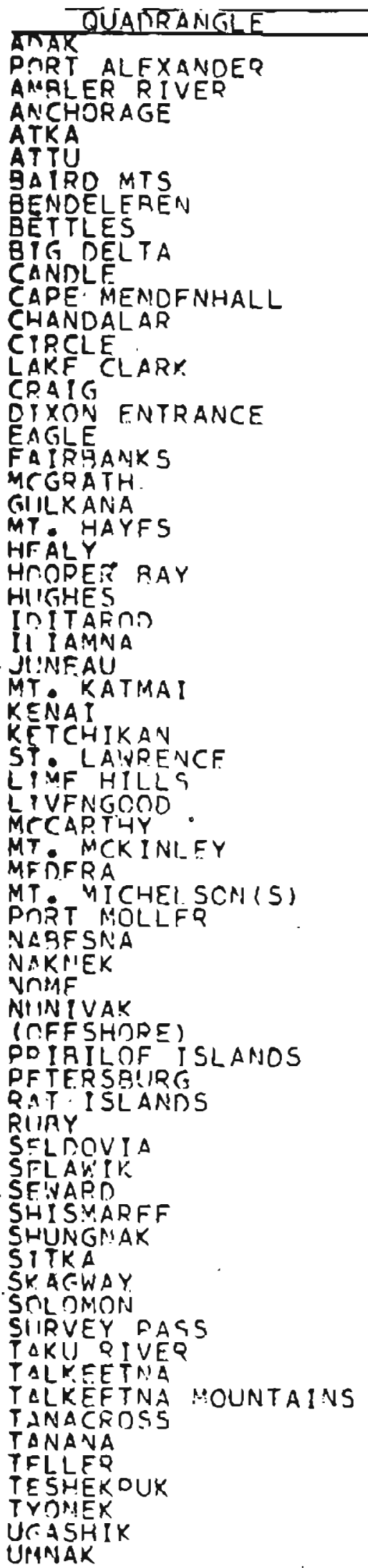

ACAK

ALEXD

A.MBR I

$\triangle A C H$

ATKA

ATTU

BAIPR

GENDE

SIG

CANINL

CAPMN

CHANR

CIRCL

CRAIG

DIXON

EAJLE

FAIR

GRATH

GULK

HAYF

HE HLY

HOJDR

HUGLS

ILITAM

JUYFU

KATNII

KEVAI

KETCH

LAWPC

L? ME

I JEN

MCAET

MC IA

MCIOA

MI $64 \mathrm{HL}$

NA BFS

NK JEK

NO AF

NUYVK

DF $=54$

DR I

PT $3 R G$

QATIS

RU'3Y

SE. - ?

SENRO

SHISF

SHIJP!

SI TKA

Skirs

SO M

TA!!!

YA: $K$

TA!KM

TAJÁC

TA!JAN

TEI ERR

TY JEY

UG $j .4 K$

UM'JAK

\begin{tabular}{ll}
\hline UPIALAKLEET & UNLATK \\
VALOEZ & VALDZZ \\
WISEMAN & WISE
\end{tabular}

DATF TYPE

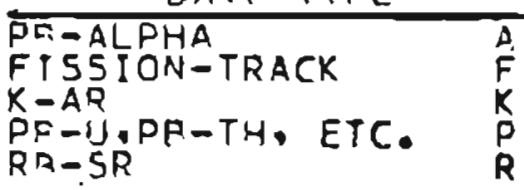

M INEqAL Dateo

ATTINCLITF

ANPHIEOLL

APATITE

AMORTHOCLLASE

BTOIITF

CLLSRITE

FELDSPAR

GLASS

TELAUCOPHANF.

HIR MAL ENIDF

I SOCHRON

MiCA

MIISCOVITE

PARAGONI ITE.

PHEMIITF.

PLAGIOCLASE

PYROXFNF

RIEBECXITF

SANITINF

SERICITF

SPHENE

WHOLE ROCK

ZIPCON!

ROCK TYP̈FS

\begin{tabular}{|c|c|}
\hline $\begin{array}{l}\text { AOAM } \\
\text { AGNS } \\
\text { ALSK } \\
\text { AWPH } \\
\text { AND } \\
\text { ANOR } \\
\text { APL } \\
\text { RAS } \\
\text { BIOT } \\
\text { DAC } \\
\text { DI } \\
\text { DIA } \\
\text { DUN } \\
\text { ECLE } \\
\text { FELS } \\
\text { GAB } \\
\text { GEI } \\
\text { GLASS } \\
\text { GNLT } \\
\text { GNS } \\
\text { GNST } \\
\text { GR } \\
\text { GPTD } \\
\text { GSCH }\end{array}$ & 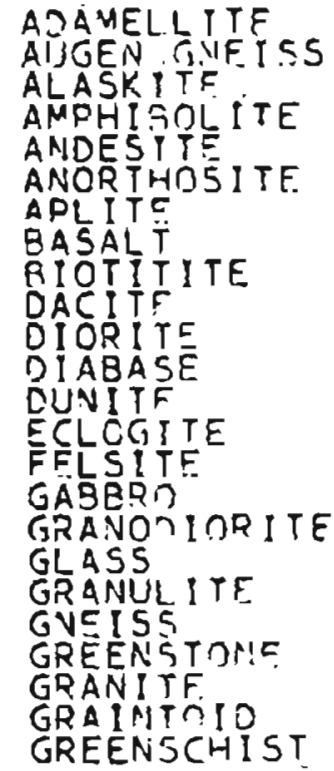 \\
\hline
\end{tabular}


Table 4. Compilation glossary (cont.)
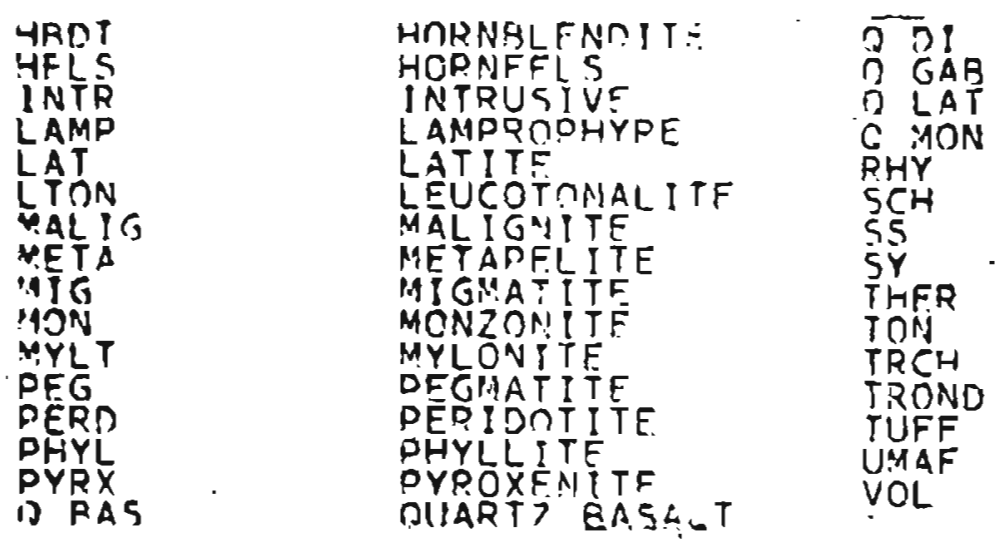
REPERENCES POR SPECIAL REPORT 10 AND OPEN-FILE REPORTS 82-86.



